appendix, a good summary of the fundamental results of probability theory and of complex analysis that are frequently used in the book.

The preceeding remarks indicate the relevance of the book for those concerned with the kind of stochastic problems that find continued practical applications. But a further point, namely the suitability of the book as a text for instruction, should be raised. This book does not appear to be designed as an introductory text in the theory of stochastic processes. It gives no hint of the present development of the theory along measure-theoretic lines in close association with potential theory. But on the other hand, it does an excellent job of maintaining in the foreground the intuitive motivation and the possible application of the theory. In the reviewer's opinion, the book would be best for students who are already familiar with a standard course in probability and an introduction to stochastic processes.

The only criticism that the reviewer can make is that the style of writing, by excessive conciseness, makes some proofs difficult to follow.

\title{
R. RESTREPO,
}

UNIVERSITY OF British COLUMBIA

Generalized Functions and Partial Differential Equations. By G. E. SHILOv. Gordon and Breach, New York (1968). xii +345 pp. U.S. $\$ 21(20 \%$ discount for payment in advance).

Part I: Generalized functions. Here the standard elementary theory of generalized functions (i.e. Schwartz distributions) is presented. Some special topics to be used in the second part are included.

Part II: Partial differential equations. Two particular problems are discussed in detail, for operators with constant coefficients: (a) Ellipticity, and especially hypoellipticity; (b) Well posed boundary problems in a half-space.

This book is elementary in the sense that only standard results from functional analysis and integration theory are used, and no previous knowledge of differential equations is assumed. It would be suitable for a first graduate course; in fact most graduate students could read it on their own. There are numerous well chosen exercises to make the book easier and more pleasant to work with. The text is clear and elegant, and provides an excellent introduction to contemporary work in partial differential equations.

\section{Colin Clark,} 8 - C.M.B.

UNIVERSITY OF BRITISH COLUMBIA 